\title{
MEASUREMENT OF THE LIFETIME OF HADRONS CONTAINING THE b-QUARK
}

\author{
J.-M. BROM a , S. ABACHI ${ }^{\text {b }}$, C. AKERLOF ${ }^{\text {c }}$, P. BARINGER ${ }^{\text {b }}$, D. BLOCKUS ${ }^{\text {a }}$, B. BRABSON ${ }^{\text {a }}$,

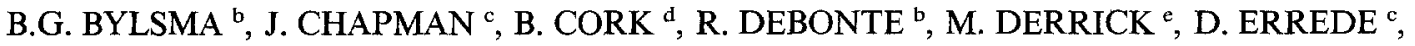 \\ K.K. GAN ${ }^{\mathrm{e}, 1}$, C. JUNG ${ }^{\mathrm{a}, 1}$, M.T. KEN ${ }^{\mathrm{c}}$, D. KOLTICK ${ }^{\mathrm{b}}$, P. KOOIJMAN ${ }^{\mathrm{e}}$, F.J. LOEFFLER ${ }^{\mathrm{b}}$, \\ J.S. LOOS ${ }^{\mathrm{e}, 2}$, E.H. LOW ${ }^{\mathrm{b}}$, R.L. McILWAIN ${ }^{\mathrm{b}}$, D.I. MEYER ${ }^{\mathrm{c}}$, D.H. MILLER ${ }^{\mathrm{b}}$, B. MUSGRAVE ${ }^{\mathrm{e}}$, \\ C.R. NG ${ }^{b}$, D. NITZ ${ }^{\text {c }}$, H. OGREN ${ }^{a}$, H.W. PAIK ${ }^{a}$, L.E. PRICE ${ }^{\text {e }}$, L.K. RANGAN ${ }^{\text {b.3 }}{ }^{\text {, J. REPOND }}{ }^{\text {, }}$,

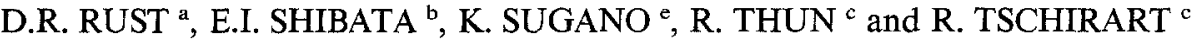 \\ a Indiana University, Bloomington, IN 47405, USA \\ ' Purdue University, West Lafayette, IN 47907, USA \\ c University of Michigan, Ann Arbor, MI 48109, USA \\ ¿ Lawrence Berkeley Laboratory, Berkeley, CA 94720, USA \\ - Argonne Nattonal Laboratory, Argonne, IL 60439, USA
}

Received 16 April 1987

Data collected with the High Resolution Spectrometer at PEP, corresponding to an integrated luminosity of $200 \mathrm{pb}^{-1}$ of $\mathrm{e}^{+} \mathrm{e}^{-}$ annihilation at $29 \mathrm{GeV}$, were used to measure the mean lifetıme of hadrons containing the bottom quark. The lifetıme was determined using the impact parameter method on events containing a high-transverse-momentum electron The result, based on a sample of 301 events, is $\tau_{\mathbf{B}}=102 \pm_{039}^{04} \mathrm{ps}$, where the indicated errors include both statistics and systematics

In the context of the standard model, the lifetime of hadrons contanining a bottom quark is determined by the electroweak coupling between the $b$ and the $\mathrm{c}$ and $\mathrm{u}$ quarks. In the Kobayashi-Maskawa scheme, the lifetime constrains the values of the matrix elements $\left|V_{\mathrm{bc}}\right|$ and $\left|V_{\mathrm{bu}}\right|[1]$. This letter presents a measurement of the lifetime of a mixture of hadrons containing the b-quark. The data sample consists of $\mathrm{e}^{+} \mathrm{e}^{-} \rightarrow \mathrm{b} \bar{b}$ annihilations at $\sqrt{s}=29 \mathrm{GeV}$, tagged by a high- $P_{\mathrm{T}}$ electron coming from the semileptonic decay of the b-quark. Two different analysis techniques are used, but the quoted result is based on a maxımum likelihood fit to the measured impact parameter distribution.

A detailed description of the High Resolution Spectrometer can be found elsewhere [2]. For this analysis, the tracking system is essential. It consists

Present address. Stanford Linear Accelerator Center, Stanford, CA 94305, USA.

2 Present address' Bell Laboratories, Naperville, IL 60566, USA

3 Present address Lockheed Missiles and Space Co, Sunnyvale, CA 94086, USA. of three coaxial components. The outer drift chamber (ODC) is made of two layers of drift tubes located at a radius of $1.9 \mathrm{~m}$, with the wires aligned parallel to the $\mathrm{e}^{+} \mathrm{e}^{-}$beam direction. The central drift chamber consists of 15 layers, 7 of which are axial, and 8 are at stereo angles of $\pm 60 \mathrm{mrad}$. It covers the radial distance between 0.2 and $1.0 \mathrm{~m}$ from the interaction point. Both of these chambers have an intrinsic resolution of about $200 \mu \mathrm{m}$. The vertex chamber [3], which is essential to the present measurement, is made of four cylindrical layers of 352 aluminized mylar tubes located at radii between 0.09 and 0.11 from the beam axis. The vertex chamber has an intrinsic resolution of $100 \mu \mathrm{m}$. All three chambers operate in the solenoidal field of $1.62 \mathrm{~T}$. The momentum resolution for tracks at large polar angles is $\sigma_{p} / p=2 \times 10^{-3} p(\mathrm{GeV} / c)$.

The tracking system is surrounded by a barrel shower counter system (BSC) divided azimuthally in 40 modules, each consisting of two sections of 3 and 8 radiation lengths, respectively, of lead-scintıllator sandwich with one radiation length sampling. 
A single layer of proportional wire chambers (PWC) 1s located between the radiator sections and is used for the localization of showers. The scintıllator signals from each section are read out by two phototubes, one at each end of the $3.68 \mathrm{~m}$ long modules. The energy resolution is $\sigma^{2}{ }_{E} / E^{2}=0.16^{2} / E+0.06^{2}(E$ in $\mathrm{GeV}$ ).

The data used for this analysis correspond to an integrated luminosity of $200 \mathrm{pb}^{-1}$ obtained at the PEP storage ring at a center of mass energy of 29 $\mathrm{GeV}$. The hadronic sample was chosen by a series of simple cuts [4], in particular, the events were required to have more than four charged particles, each of which had to register in at least 9 of the 17 layers of the outer and central drift chambers. These cuts removed Bhabha scattering and muon pairs. To ensure a clean electron identification, the events used were in the central region of the detector, defined by requiring $|\cos \theta| \leqslant 0.7$ where $\theta$ is the angle between the thrust axis of the event and the beam axis.

Electrons were identified by associating energy deposited in the BSC with the reconstructed track. This was done by first selecting tracks that have one 1solated cluster in the PWC within $\pm 5 \mathrm{~cm}$ of the projection of the reconstructed track. The energy deposited $(E)$ was then determined and compared to the momentum of the charged particle $(P)$. The distribution of this ratio is shown in fig. 1 and a clear peak at $E / P=1$ indicates the electrons in the sample. The background from photon conversion was negligibly small because a very small amount of material (less than $0.01 X_{0}$ ) precedes the tracking system. The final sample was then obtained by selecting particles with $0.75 \leqslant E / P \leqslant 1.33$. The further requirements that $P$ exceed $2.0 \mathrm{GeV} / c$ and the transverse momentum with respect to the thrust axis $P_{\mathrm{T}}$ exceed $1.1 \mathrm{GeV} / c$ were imposed [5]. Events where the electron candidate shared cells in the vertex chamber with any other track were rejected.

From a Monte Carlo (MC) generation of the $\mathrm{e}^{+} \mathrm{e}^{-} \rightarrow \mathrm{q} \overline{\mathrm{q}}$ process [6] and a detailed simulation of the detector, the data sample resulting from these cuts is estimated to contain $(53 \pm 7) \%$ of $\mathrm{b} \bar{b},(22 \pm 3) \%$ of $\mathrm{c} \overline{\mathrm{c}}$, and $(25 \pm 5) \%$ of other final states. The latter, which are mainly due to hadrons misidentified as electrons, constitute the background under the peak at $E / P$ in fig. 1 . The background also includes a small fraction of light quark annihilation events. The events coming from the decay $b \rightarrow c \rightarrow e$, which are mainly removed by the $P_{\mathrm{T}}$ cut, are considered part of the $\mathrm{b} \overline{\mathrm{b}}$ sub-sample. The event breakdown is similar to those observed by other groups who have measured the semileptonic branching ratios of the $b$ and $c$ quarks [7]. Tau pairs are eliminated by the combined electron identification and multiplicity $(>4)$ cuts.

The $\mathrm{e}^{+} \mathrm{e}^{-}$annihilation point was calculated for each data run using Bhabha scattering events. The impact parameter distribution for tracks from such events, which occur with a high rate [8], gives a beam size of $\sigma=385 \pm 10 \mu \mathrm{m}$ in the horizontal direction and $\sigma=95 \pm 10 \mu \mathrm{m}$ in the vertical direction [9]. These values represent a folding of the true beam size, the measuring error, and any beam motion during the data run.

The above selection criteria yielded a sample of 312 high- $P_{T}$ electrons, which were then used to determine the impact parameter distribution. The impact parameter $(\delta)$ is defined in the plane perpendicular to the beams as the distance of closest approach between the interaction point and the projection of the reconstructed electron trajectory. The value of $\delta$ is positive (negative) if the projected track intersects the b-hadron flight path, represented by the thrust axis, in front of (behind) the $\mathrm{e}^{+} \mathrm{e}^{-}$interaction point. The error on the calculated impact parameter for these electrons ranges from 200 to 550 $\mu \mathrm{m}$ depending on the azimuthal orientation of the events. Fig. 2 shows the distribution of the errors. The peak around $500 \mu \mathrm{m}$ comes from the larger beam size in the horizontal direction. The distribution of impact parameters is shown in fig. 3 for the 301 tracks that have $|\delta|$ less than $2.0 \mathrm{~mm}$. The mean of the impact parameter distribution is $80 \pm 27 \mu \mathrm{m}$.

The lifetime was determined by using a maximum likelihood technique. The probability density function for the impact parameter distribution was calculated from events generated by the Monte Carlo technique that were processed in the same way as the data. Three samples of $\mathrm{e}^{+} \mathrm{e}^{-} \rightarrow \mathrm{b} \bar{b}$ events were generated with lifetimes of $0.0,1.0$, and 7.0 ps. For each MC sample, the generated impact parameter distr1bution was extracted, ignoring experimental resolut1on effects. Each distribution has an exponential tail and its mean value scales linearly with the generated lifetıme. This impact parameter results from com- 


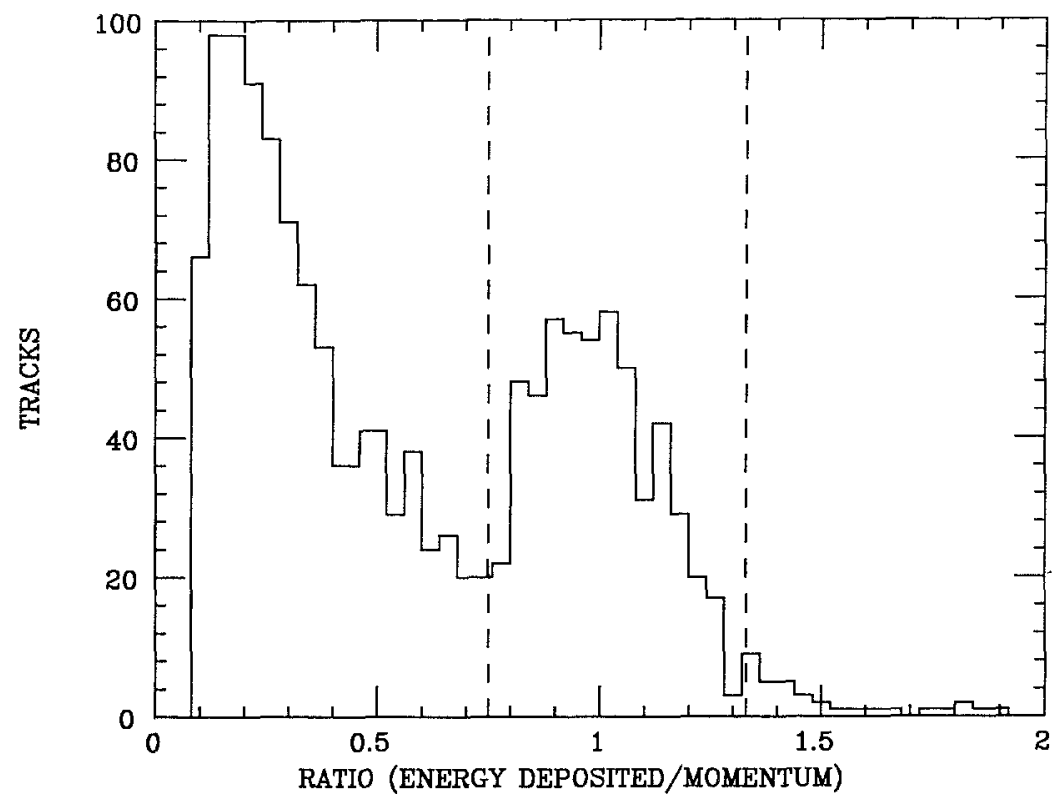

Fig. 1. Distribution in $E / P$ ratio for all particles with isolated clusters in the BSC and with a minımum energy deposit of $200 \mathrm{MeV}$

bination of the lifetime of the b-hadron (exponential shape), the b-hadron momentum and the leptonic decay kinematics. Although this momentum is not directly measurable it is possible to use the momentum averaged distribution obtained from the Monte-

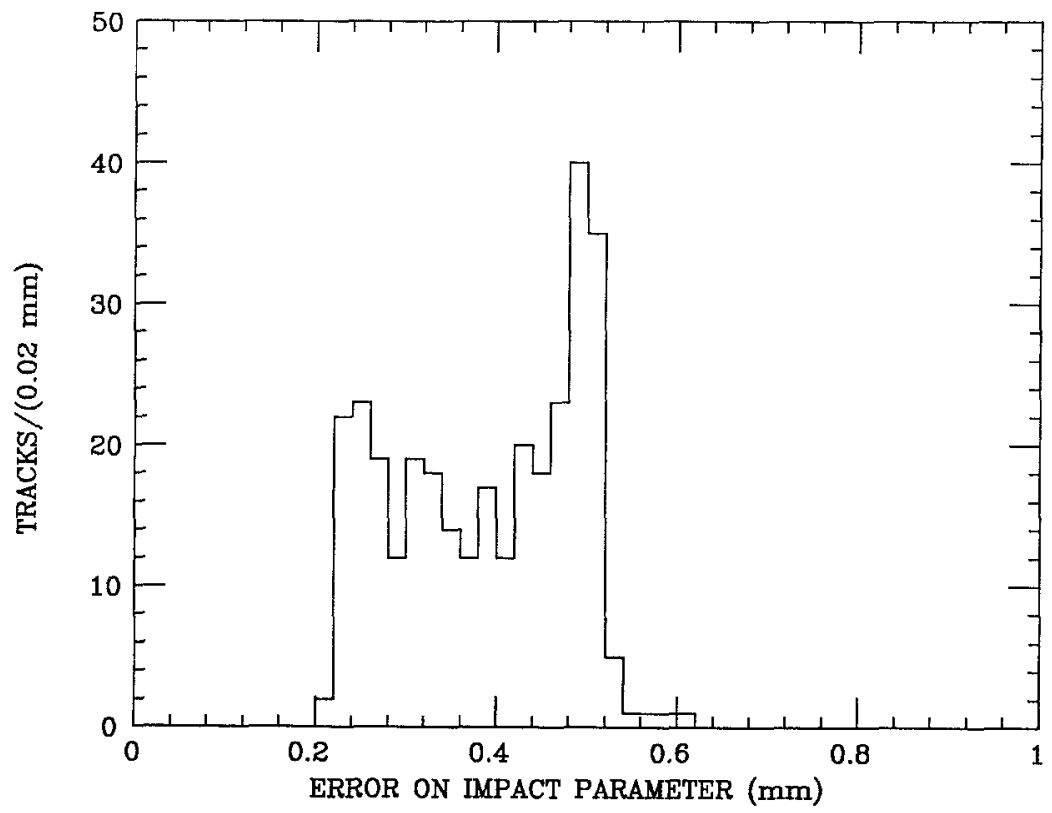

Fig. 2. Error distrıbution for the impact parameter of tagged electrons. The higher values are domınated by the larger dimension of the beam in the horizontal plane. 


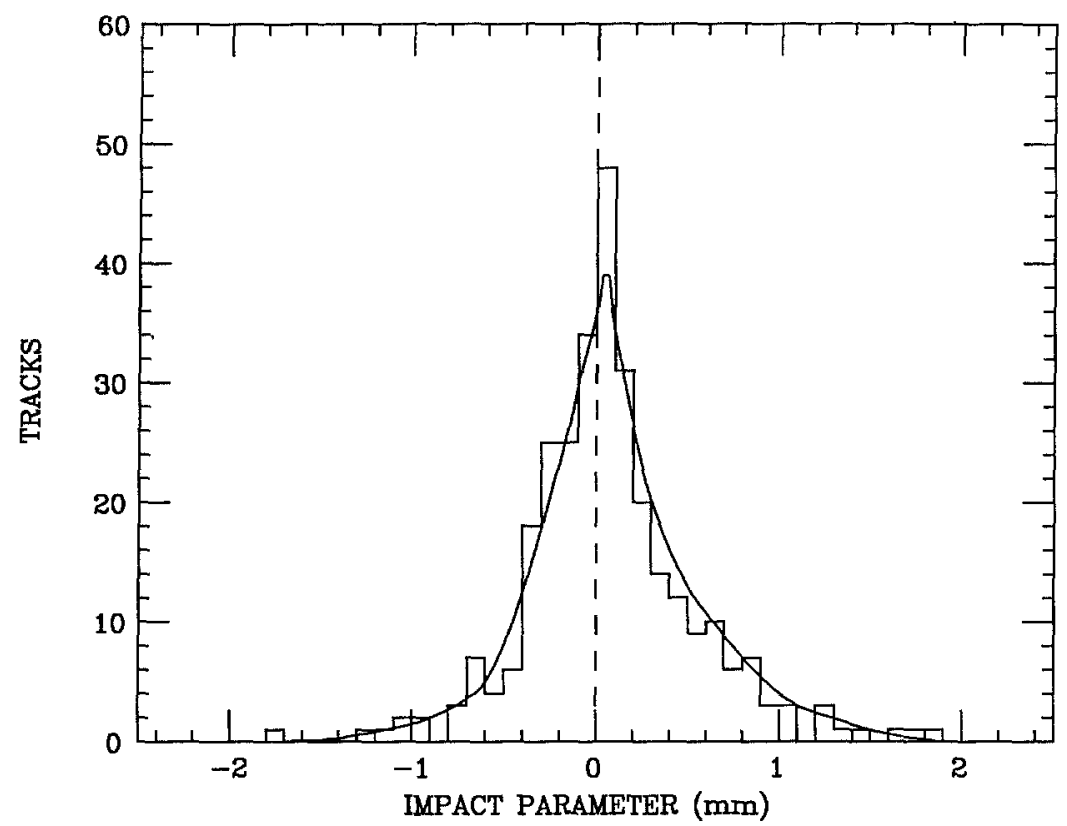

Fig 3 Impact parameter distribution for tagged electrons. The solid curve represents the result of the fit described in the text.

Carlo ${ }^{\# 1}[9]$. The probability function was then calculated using the form

$\frac{1}{\alpha c \tau_{\mathrm{B}}} \iint \exp \left(\frac{-\delta}{\alpha c \tau_{\mathrm{B}} \sqrt{\gamma^{2}-1}}\right) \frac{1}{\sqrt{\gamma^{2}-1}} P(\gamma) \mathrm{d} \gamma \mathrm{d} \delta$

where $\tau_{\mathrm{B}}$ represents the proper lifetime of the particle, $\delta$ is the impact parameter, $P(\gamma)$ is a function representing the distribution in gamma of the b-hadron, and $\alpha$ is the angular factor that relates the decay length to the impact distance of the electron. From the Monte Carlo, the value of $\alpha$ was estimated to be $0.40 \pm 0.08$. The distribution $P(\gamma)$ was checked to be independent of the generated lifetime and was well fitted by a gaussian function whose parameters depend on the three components (i.e. b-hadrons, chadrons and background). In order to check the correctness of this analysis, this method was used to determine the generated lifetime of the three Monte Carlo samples. The results of this likelihood analysis were $0.2 \pm 0.1,1.1 \pm 0.1$, and $5.7 \pm 1.5$ ps, yielding good agreement with the generated values of $0,1.0$ and 7.0 ps. Fig. 4 shows the result of the fit on the

\#! The b-hadron momentum is peaked around ( $x=080 \mathrm{pm} 0.03$ ) so the averaging is over a small range in momentum. See, for instance, ref [10]. generated impact parameter for a Monte Carlo subsample (1 ps lifetıme).

To construct the final probability density function, this primary distribution was then convoluted with a gaussian representing the experimental resolution. The latter was centered on the measured $\delta$ value and with a width equal to the calculated experimental error. The overall probability density function is then the sum of three distributions of this form representing sources of b-events, c-events, and background, weighted by the relative proportion of each contribution. From the fit to the data, which includes all leptons with $|\delta| \leqslant 2.0 \mathrm{~mm}$, the lifetime of hadrons containing a bottom quark is measured to be $\tau_{\mathrm{B}}=0.96 \pm 030 \mathrm{ps}$.

The uncertainty in the impact parameter calculation depends on the errors that are assigned to the measured track parameters and so directly influences the final results of this analysis. To check whether this error assignment is proper, a twodimensional maximum likelıhood fit was performed with the addition of a scaling factor multiplying the calculated tracking error, which appears as the width of the gaussian representing the experimental resolution. The result of that new fit was $\tau_{\mathrm{B}}=$ $1.02 \pm_{036}^{039} \mathrm{ps}$, consistent with the result found in the 


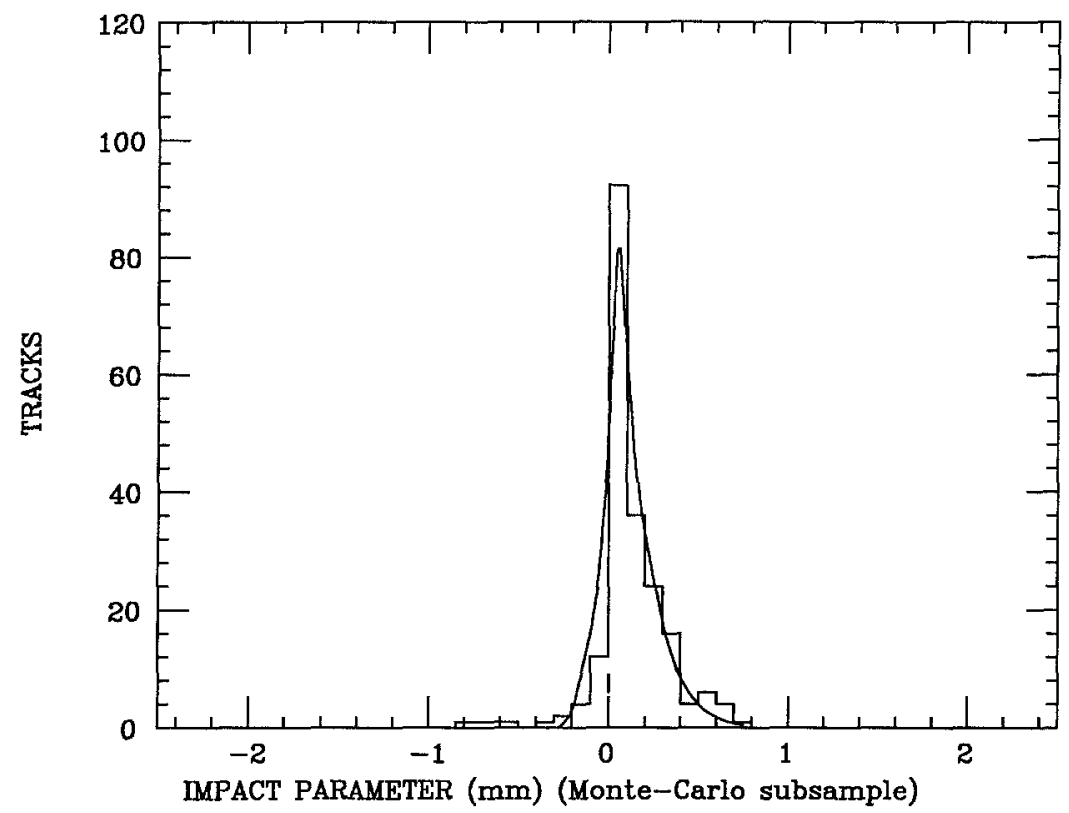

Fig. 4. Generated impact of Monte Carlo event, without experimental resolution effects. The solid curve is the result of the fit.

previous analysis. The new fit scaled up the calculated tracking error by a factor of $1.18 \pm 0.06$. However, the departure of the error scaling factor from a value of 1.0 is one indication that the fitted lifetime error contains a non-negligible part due to systematics. This effect arises from the correlation between the fitted lifetime value and the experimental resolution error scaling factor.

Additional uncertainties in the estimation of the mean value of the generated impact parameter distributions for the background and for the c-quark mesons will affect the $b$ quark lifetıme. However, the effect is small: changing the average c-meson lifetime by \pm 0.2 ps changes the b-lifetıme by \pm 0.04 ps and changing the background lifetime by 0.1 ps results in a negligible change to the b-lifetıme. Reducing the relative proportion of $b$-events in the sample from 0.53 to 0.46 changes the lifetime by $0.06 \mathrm{ps}$. The uncertainty due to the factor $\alpha$ is estimated to be \pm 0.15 ps. Adding these additional systematic contributions in quadrature to the above error gives a total error (including both statistical and systematic effects) of $\pm_{039}^{042}$.

As a check of the analysis, the b-lifetime was measured using the weighted mean technique [11]. Each measured value of the impact parameter was weighted by $1 / \sigma^{2}$, where $\sigma$ represents the error on the measured value. From the Monte Carlo samples the respective contributions to that weighted mean are: $154 \mu \mathrm{m}$ for b-events (generated with a lifetime of $1.0 \mathrm{ps}$ ), $54 \mu \mathrm{m}$ for c-events, and $5 \mu \mathrm{m}$ for the background events. From this analysis, the weighted mean value for the impact parameter distribution is $84 \pm 28 \mu \mathrm{m}$, corresponding to a lifetime of $0.92 \pm 0.37$ ps, including the error in connecting impact parameter to lifetime. This is in excellent agreement with the value measured using the maximum likelihood technique. Because of the lack of precision of this method, which is more sensitive to the shape of the distributions, this second analysis was used only as a cross-check.

In conclusion, the value of the lifetıme containing a bottom quark is $\tau_{\mathbf{B}}=1.02 \pm_{039}^{042}$ ps. This is in good agreement with results from other experiments (fig. 5 ). This measurement represents an average over all of the hadrons containing a b-quark that are produced in $\mathrm{e}^{+} \mathrm{e}^{-}$annihilation at $29 \mathrm{GeV}$. If the spectator diagrams dominate such decays, then the result will be close to the lifetime of the b-quark itself and so can be used to evaluate the $\left|V_{\mathrm{bu}}\right|$ and $\left|V_{\mathrm{bc}}\right|$ elements of the Kobayashi-Maskawa matrix. If the bquark does not mix with lower mass quarks, the b- 


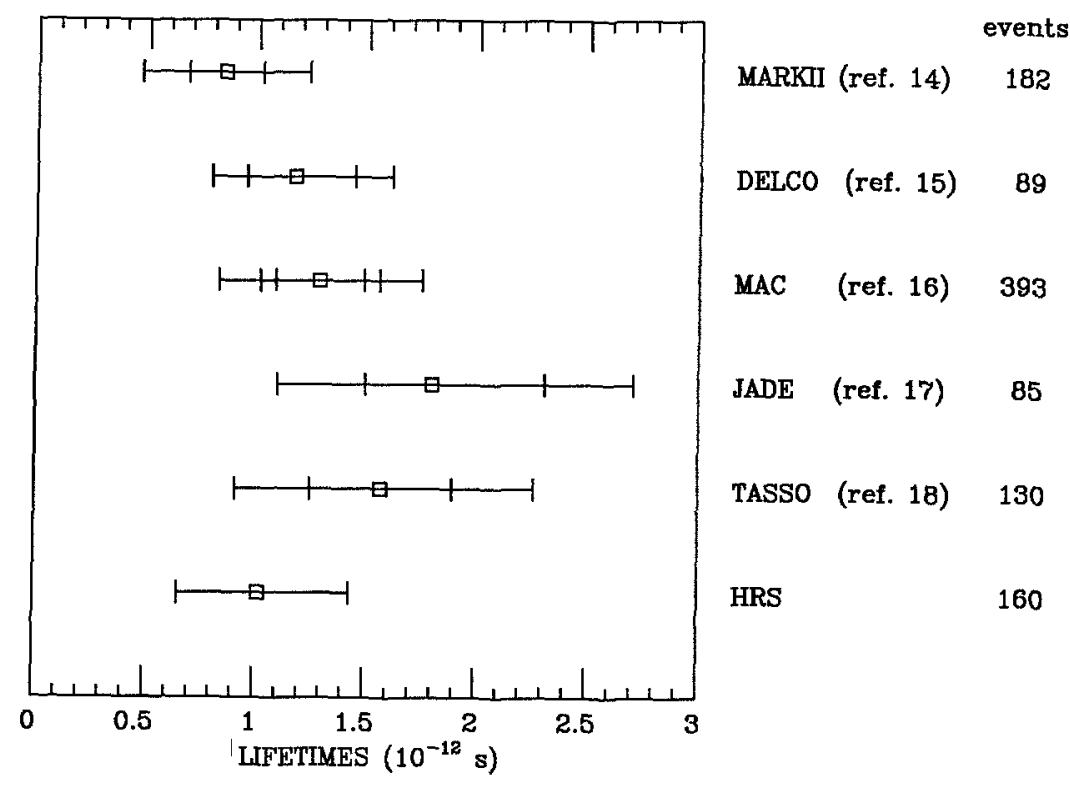

Fig 5. Comparison of most recent results on the B-lifetıme. To compare the different results, systematic and statistical errors have been added. The number of events takes into account the purity of the samples.

quark should be stable. Thus, a low value for the lifetime implies relatively large coupling values. This can be quantified using the following formula [12]:

$\tau_{\mathrm{B}}=\frac{\mathrm{BR}(\mathrm{b} \rightarrow \mathrm{Xe}) \times 10^{-14}}{0.58\left|\mathrm{~V}_{\mathrm{bc}}\right|^{2}+1.18\left|V_{\mathrm{bu}}\right|^{2}} \mathrm{~s}$.

Using $(10.80 \pm 0.08) \%$ [5] for the semileptonic branching ratio of the b-quark, $\left|V_{\mathrm{bu}}\right|=0.46\left|V_{\mathrm{bc}}\right|$ [13], and $\tau_{\mathrm{B}}=1.02 \pm_{037}^{041} \mathrm{ps}$, the above equation yields

$\left|V_{\mathrm{bc}}\right|=0.036 \pm 0006$

We are grateful to the cryogenic group of SLAC and to the technical staffs of PEP, and the collaborating institutions, without whom this experiment would not have been possible. This work was supported in part by the US Department of Energy under Contracts W-31-109-ENG-38, DE-AC0276ER01112, DE-AC03-76F00098, DE-AC0276ER01428, and DE-AC02-84ER40125.

\section{References}

[1] L L. Chau, Phys Rep. 95 (1983) 1

[2] HRS Collab, D. Bender et al., Phys. Rev D 30 (1984) 515
[3] P Baringer et al, preprint IUHEE-80 (Indiana University), submitted to Nucl Instrum. Methods.

[4] HRS Collab , D. Bender et al., Phys. Rev. D 30 (1985) 1.

[5] R.Ng, Ph.D Thesis, Purdue University (1987). unpublished

[6] B. Andersson et al., Phys Rep 97 (1983) 32.

[7] See e.g E Fernandez et al., Phys. Rev. Lett. 50 (1983) 2054

[8] HRS Collab., M. Derrick et al., Phys Rev. D 34 (1986) 3286.

[9] HRS Collab., C. Jung et al , Phys. Rev. Lett. 56 (1986) 1775.

[ 10] J.M. Izen, Report DESY-84-104 (1984), also in Proc. Lund Multiparticle Dynamics (1984) p.727.

[11] MAC Collab., W W. Ash et al., preprint submitted to XXIII High energy physıcs Conf. (Berkeley, CA, July 1986).

[12] V. Luth, Invited talk presented Intern. Symp. on Production and decay of heavy flavour (He1delberg, May 1986), SLAC Report SLAC-PUB-4052.

[13] S. Stone, preprint CLNS-86/753, review talk presented 6th Intern. Conf. on Physics in collıson (Chıcago, IL, September 1986)

[14] MARK II Collab, N.S. Lackyer et al, Phys Rev. Lett 51 (1983) 1316

[15] DELCO Collab., DE Klem, Ph D Thesis, SLAC-300 (1986), also submitted to Phys. Rev.

[16] MAC Collab., W.W. Ash et al., Phys. Rev Lett 58 (1987) 640

[17] JADE Collab., W. Bartel et al , Report DESY 86-001 (1986). unpublished.

[18] TASSO Collab, A. Caldwell et al, paper presented XXIII High energy physics Conf. (Berkeley, CA, July 1986). 